\title{
Morphology Control and Photocatalytic Characterization of Yttrium-doped
} Hedgehog-like $\mathrm{CeO}_{2}$

\author{
Bin Xu a,b,c, Qitao Zhang a,b, Saisai Yuan ${ }^{\mathrm{a}, \mathrm{b}}$, Ming Zhang a,c *,Teruhisa Ohno ${ }^{\mathrm{b}, \mathrm{d}, \mathrm{ef}, \text {, * }}$ \\ a School of Chemistry and Chemical Engineering, Yangzhou University, Yangzhou 225002, China \\ ${ }^{\mathrm{b}}$ Department of Applied Chemistry, Faculty of Engineering, Kyushu Institute of Technology, Kitakyushu 804-8550, \\ Japan \\ c Test Center, Yangzhou University, Yangzhou 225002, China \\ d JST, PRESTO and ACT-C, 4-1-8 Honcho Kawaguchi, Saitama 332-0012, Japan \\ e JST,ACT-C,4-1-8 Honcho Kawaguchi, Saitama 332-0012, Japan \\ ${ }^{\mathrm{f}}$ Research Center for Advanced Eco-fitting Technology, Kyushu Institute of Technology, Tobata, Kitakyushu \\ 804-8550, Japan \\ *corresponding author. Tel: +8193884 3318; Fax: +81938843318 \\ E-mail address: 1xyzhangm@yzu.edu.cn (Ming Zhang) \\ tohno@.che.kyutech.jp (T.Ohno)
}

\begin{abstract}
Yttrium-doped hedgehog-like ceria with a high concentration of oxygen vacancies was successfully prepared by a traditional hydrothermal process. Crystal phase composition, morphology, and size of as-fabricated products were found to be greatly dependent on the mole ratio of original materials and reaction temperature. The morphology and size of the as-prepared samples were characterized by FE-SEM, HR-TEM and HADDF-STEM. Structure information was obtained by XRD and Raman analyses. Absorption band and band gap energy, which are responsible for the observed photocatalytic behavior, were investigated by UV-vis diffuse reflectance. In addition, the optimum experimental conditions for target products were determined. The as-prepared doped three dimensional (3D) hierarchical structure samples showed higher photocatalytic activity than that of one dimensional (1D) octahedral and nanorod morphology ceria as evaluated by measuring $\mathrm{CO}_{2}$ liberation form the photocatalytic decomposition of acetaldehyde.
\end{abstract}

Keywords: yttrium-doped ceria, hierarchical structure, oxygen vacancy, morphology control, photocatalytic activity

\section{Introduction}

Cerium dioxide, an important rare-earth oxide, has attracted much attention due to its significant fluorite-type structure, remarkable redox properties [1] and prominent oxygen storage and release capacity (OSC) via facile conversion between $\mathrm{Ce}^{4+}$ and $\mathrm{Ce}^{3+}$ oxidation states [2]. It has been extensively utilized in many practical applications such as polishing materials [3], solar cells[4], ultraviolet blocking materials $[5,6]$ and photocatalytic materials. Pure $\mathrm{CeO}_{2}$, an n-type semiconductor, has a band gap $(3.2 \mathrm{eV})$ similar to those of other commonly utilized semiconductor-based photocatalyst such as $\mathrm{TiO}_{2}$ [7] and possesses potential as a suitable photocatalyst. Recently, $\mathrm{CeO}_{2}$ materials with different morphologies have been synthesized by various methods including thermal evaporation [8], coprecipitation [9], and the sol-gel technique [10]. Previous studies have indicated that excellent catalytical performance and easy 
functionalization of $\mathrm{CeO}_{2}$ materials can been achieved by controlling their structural properties [11]. For instance, our group successfully fabricated sesame ball-like $\mathrm{CeO}_{2}: \mathrm{Y} 3+/ \mathrm{P}(\mathrm{St}-\mathrm{AA})$ composite microspheres [12]. Compared with conventional nanowires, nanorods and nanocubes as well as nanoparticles with other shapes, newly constructed $\mathrm{CeO}_{2}$ with a hierarchical architecture will acquire some special properties that single morphology $\mathrm{CeO}_{2}$ does not possess $[10,13,14]$. Although some progress has been made in the fabrication of hierarchical architectures composed of 1D nanostructures, the methods usually need special templates through a complex experimental course. For example, Sam L. Mitchell et al. had fabricated coral-like mesostructured $\mathrm{CeO}_{2}$ using an amino acid as template [15]. Zhong et al. reported the preparation of 3D flowerlike ceria at 180 ${ }^{0} \mathrm{C}$ with $\mathrm{TBAB}$ as a surfactant and $\mathrm{EG}$ as a solvent [16].

The use for morphology and size-controlled metal and metal oxide composites and different ionic doping are the three most commonly utilized methods for enhancing the photocatalytic properties of $\mathrm{CeO}_{2}$-based materials. The keypoint in these approaches is changing the oxygen vacancy concentrations of products, because oxygen vacancies can act as electron or hole capture centers and can trap the photogenerated electrons or holes excited by ultraviolet or visible light. Further oxygen vacancies can effectively restrain the recombination of electron-hole pairs, resulting in improvement of photocatalytic activities [17]. Application of more than two or all of the above methods to the preparation of a 3D hierarchical structure of $\mathrm{CeO}_{2}$ with a high concentration of oxygen vacancies would be a huge challenge. To the best of our knowledge, there has been no report about yttrium-doped hedgehog-like $\mathrm{CeO}_{2}$ obtained by facile controlled synthesis.

Herein, we report for the first time a facile and feasible approach to prepare $\mathrm{CeO}_{2}$ with a yttrium-doped hedgehog-like hierarchical structure by a simple template-free hydrothermal technique using the simple inorganic salts $\mathrm{Ce}\left(\mathrm{NO}_{3}\right)_{3}, \mathrm{Y}\left(\mathrm{NO}_{3}\right)_{3}$ and $\mathrm{Na}_{3} \mathrm{PO}_{4}$ as original materials. The morphology of as-fabricated samples can be controlled by tuning the reaction temperature and reactant concentration.

\section{Experimental section}

\subsection{Preparation.}

$\mathrm{CeO}_{2}$ with yttrium-doped hedgehog-like hierarchical structure was fabricated through the traditional hydrothermal process. All original analytical grade materials were purchased from Wako Co., Ltd and were used without any further purification. Cerium nitrate $\left(\mathrm{Ce}\left(\mathrm{NO}_{3}\right)_{3} \cdot 6 \mathrm{H}_{2} \mathrm{O}\right)$

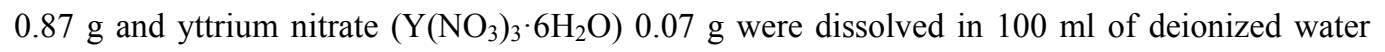
with vigorous magnetic stirring for 30 minutes at room temperature. Then $7.7 \mathrm{mg}$ sodium phosphate $\left(\mathrm{Na}_{3} \mathrm{PO}_{4}\right)$ was added to the aqueous solution. The mixture was stirred continuously for one hour. The mixed solution was then sealed in a Teflon-lined autoclave and heated at 100 250 ${ }^{0} \mathrm{C}$ for 12-96 hours. After cooling to room temperature, a light yellow precipitate was collected by centrifugation and washed several times with deionized water and ethanol. Target products were obtained after dried in the air at $60{ }^{\circ} \mathrm{C}$ for one day.

\subsection{Characterizations.}

An X-Ray diffractometer (XRD) ( Bruker-AXS, D8 sss) with $\mathrm{Cu}$ Ka radiation $(\lambda=1.5406 \AA$ ) and monochromator was used to identify the crystalline phase. The crystallite parameters and size were calculated by the Rietveld method (TOPAS 4.0). $\mathrm{N}_{2}$ adsorption and desorption isotherms were recorded at $77 \mathrm{~K}$ using a Nova $4200 \mathrm{e}$ instrument. The samples were precisely weighted and 
degased at $373 \mathrm{~K}$ for $3 \mathrm{~h}$. The specific surface area was calculated by the 5 points Brunauer-Emmit-Teller theory. Raman analysis (Renishaw, In via) was performed using a $532 \mathrm{~nm}$ excitation laser with $5 \mathrm{~mW}$ and an air-cooled CCD detector. Raman peak shifts were determined by fitting with the Lorentzian and Gaussian composite function. Morphology and size of as-fabricated products were examined by using a field emission scanning electron microscope (FESEM) (Hitachi, S-4800, 15KV), high-resolution transmission electron microscope (HRTEM) and HADDF-STEM (FEI, Tecnai G2 F30 S-TWIN, 300KV). Inductively coupled plasma atomic emission spectrum (ICP-AES) (Shimadzu, ICPS-8000) was used to identify the yttrium's stability in the solution.

\subsection{Photocatalytic Evaluations.}

UV lighter using black light (UVP, XX-15BLB) can be used in order to remove possible organic materials adsorbed on the surface of samples more than one week before evaluation of photocatalytic activity. The photocatalytic activity of as-fabricated samples was assessed by ability for decomposition of acetaldehyde. Twenty milligrams of powder was spread on the bottom of a glass dish, and the glass dish was placed in a Tedlar bag (AS ONE Co. Ltd.). Five hundred ppm of acetaldehyde was injected into the bag together with $125 \mathrm{~cm}^{3}$ of artificial air. Then the bag, which was fabricated above, was put into a dark place at room temperature for 2 hours for the purpose of reaching an adsorption equilibrium A light-emitting diode (LED; Epitex, L365), which emitted light at wavelengths of ca. $365 \mathrm{~nm}$, was used as a light source, and its intensity was controlled at $0.9 \mathrm{~mW} \mathrm{~cm}{ }^{-2}$. The concentration of generated $\mathrm{CO}_{2}$ as s function of irradiation time was monitored by a gas chromatography (Shimadzu GC-8A, FID detector) equipped with a Porapak N-packed column and a methanizer (GL Science, MT-221).

\section{Results and discussion}

\subsection{Structure characterization}

Typical X-ray diffractometer (XRD) patterns of samples obtained under different conditions are shown in Fig. 1 (A)-(D). All of the diffraction lines could be indexed to the phase of ceria with a cubic fluorite structure (JCPDS No. 43-1002). The sharp diffraction peaks from all samples suggest a high degree of crystallinity of fabricated samples, and no impurity peaks appeared, indicating that reaction time, amount of the mineralizer $\left(\mathrm{Na}_{3} \mathrm{PO}_{4}\right)$ and concentration of the dopant (yttrium nitrate) have no effect on crystalline phase purity of products. The relative intensity of each peak does not change, suggesting that there is no preferred orientation. A weak peak at $2 \theta=31.38^{0}$ assigned to $\mathrm{Ce}\left(\mathrm{PO}_{4}\right)(111)$ can be seen in the pattern shown in the Fig. 1(D). The results show that part of $\mathrm{Ce}^{3+}$ reacted directly with $\mathrm{PO}_{4}{ }^{3+}$ in the solution, resulting in $\mathrm{CePO}_{4}$ precipitation, instead of occur oxidation to form $\mathrm{CeO}_{2}$ owing to the low reaction temperature (below $170{ }^{\circ} \mathrm{C}$ ). With increase in temperature, impurity crystal phase cerium phosphate disappeared. This phenomenon occurs because a high temperature is beneficial for $\mathrm{Ce}^{3+}$ to $\mathrm{Ce}^{4+}$ oxidation reaction.

Rietveld refinements were also carried out according to the diffraction lines by varying parameters such as background, unit cell, and isotropic thermal parameters. When the reaction temperature and time were changed while maintaining the amount of the dopant, the variations of lattice value $(a=5.3300 \pm 0.0060 \AA)$ were smaller. However, the lattice parameter showed significant variation for as-fabricated samples with change in dopant stoichiometry. The unit cell value a changed from 5.33052 to $5.27510 \AA$ (see the Table 1). It can be clearly seen that the lattice 
value (a) after doping is smaller than the bulk $\mathrm{CeO} 2$ lattice parameter value a $=5.41048 \AA$ even though the space group is still Fm-3m before and after doping. This indicates that yttrium has partially substituted cerium ions and introduced into the interior lattice, maintaining the ceria cubic fluorite structure instead of forming a $\mathrm{Y}_{2} \mathrm{O}_{3}-\mathrm{CeO}_{2}$ composite. In addition, a linear relationship could be drawn between the lattice value and amount of doping. In other words, the amount of dopant plays a definitive role in determining ceria lattice parameters. Yttrium enters the crystal lattice and substitute cerium ions of the crystal lattice. Consequently, the crystal lattice would contract due to an increase in oxygen vacancy concentration with increase in yttrium content, despite of the fact that the ionic radius of yttrium is larger than that of cerium (1.019 and $0.97 \AA$, respectively). Particles sizes (D) of as-fabricated samples are also be obtained by Whole Pattern Profile fitting instead of single peak refinement (Scherrer formula). D values are randomly distributed between the 20 and $60 \mathrm{~nm}$. A comparison of our results with those of previous studies $[8,18,19]$ were reached the following conclusions. Although changes of particles size resulted in the crystal lattice expansion or contraction of $\mathrm{CeO}_{2}$, the effects would become weaker after yttrium doping.

Raman technology is considered to be a very efficient and nondestructive technique for characterization of ceria nanoparticles and their derivatives. Raman spectra of as-fabricated samples under various experimental conditions are shown in Fig. 2 (A)-(D). A strong Raman shift at $\sim 460 \mathrm{~cm}^{-1}$ and a relatively weak shift at $\sim 600 \mathrm{~cm}^{-1}$ can be detected. The shift at $\sim 460 \mathrm{~cm}^{-1}$ can be assigned to F2g vibration of the fluorite-type structure and it can be considered as the symmetric stretching mode of oxygen atoms around cerium ions, and the molecule retains its tetrahedral symmetry throughout. Based on the results of previous studies [20-22] and our own studies, a weak and less prominent band near $\sim 600 \mathrm{~cm}^{-1}$ can be attributed to a nondegenerate longitudinal optical mode caused by a local $\mathrm{Ce}-\mathrm{O}\left(\mathrm{R}_{\mathrm{Ce}-\mathrm{O}}\right)$ bond symmetry stretch. According to previous reports that not all cerium ions show $\mathrm{Ce}^{4+}$ chemical valence in the lattice; small cerium ions show $\mathrm{Ce}^{3+}$ tervalence. In order to maintain the particles in an electrically neutral state, the lattice oxygen would escape from the structure and finally result in the formation of intrinsic oxygen vacancies. Oxygen vacancies perturb the local Ce-O bond symmetry. A new and weak Raman shift at $\sim 530 \mathrm{~cm}^{-1}$, which cannot be detected in Raman spectra of pure $\mathrm{CeO}_{2}$, can be seen in the inset of Fig. 2 (C). It is attributed to extrinsic oxygen vacancies caused by doping ( $\mathrm{R}_{\text {dopant }}$ ). As a dopant, yttrium enters the cubic fluorite lattice of $\mathrm{CeO}_{2}$ and substituted cerium. In order to maintain electronic neutrality, doping cations showing different valence states with $\mathrm{Ce}^{4+}$ and part of the oxygen would also escape from lattice to form extrinsic oxygen vacancies. The relativity intensities of Raman shifts (F2g and $\mathrm{R}_{\text {dopant }}$ ) were calculated and are shown in the Table1, It can be seen that the value of $R_{\text {dopant }} / F 2 g$ rapidly increased at first and then reached an approximate balance with further increase in dopant content. The trend for change in the value can be attributed to amount of dopant. At the first yttrium was introduced into the bulk $\mathrm{CeO}_{2}$, and more extrinsic oxygen vacancies were obtained. Therefore, the value of $\mathrm{R}_{\mathrm{dopan}} / \mathrm{F} 2 \mathrm{~g}$ ratio rapidly increased. When excessive dopants were added, the oxygen vacancy concentration increased and eventually reached a steady plateau level in order to maintain electron neutrality of the system. This result of Raman analysis is consistent with the following activity evaluation.

\subsection{Morphology characterization}

The morphology and evolution process of as-fabricated products were investigated by 
FE-SEM and HR-TEM. Fig. 3 (a) shows a panoramic FE-SEM image of yttrium-doped $\mathrm{CeO}_{2}$ particles. It can be seen that the particles are uniform octahedral particles with diameters of approximately $200 \mathrm{~nm}$. The lattice spacing of the parallel fringes in the inset graph is $0.32 \mathrm{~nm}$, corresponding to the (111) plane of $\mathrm{FCC} \mathrm{CeO}_{2}$. In addition, the composition of elements and distribution of the as-fabricated products obtained under different experimental conditions were analyzed by STEM-HAADF images and EDS mapping images. Fig. 4 shows the existence of not only cerium but also yttrium, with yttrium ions being dispersed evenly on the $\mathrm{CeO}_{2}$ octahedral structure surface and in the bulk. Herein we can draw a conclusion that yttrium enters the lattice and dopes the parts of cerium successfully. The results for composition of elements and distribution are well consistent with the results of the XRD and Raman analysis. Fig. 3 (b) shows that with increase in the amount of $\mathrm{Na}_{3} \mathrm{PO}_{4}$, a small number of nanorods with length of $50 \mathrm{~nm}$ and diameters of $10 \mathrm{~nm}$ grew on the eight octahedral surfaces. The lattice spacing of the parallel fringes in the inset graph are $0.32 \mathrm{~nm}$ and $0.27 \mathrm{~nm}$, respectively, corresponding to the (111) plane and (200) plane of $\mathrm{FCC} \mathrm{CeO}_{2}$. The inset images demonstrates that the nanorod mainly exposed the (200) planes as well as the octahedral exposed the (111) planes. Fig. 3(c) reveals that with further increase in the amount of $\mathrm{Na}_{3} \mathrm{PO}_{4}$, many more nanorods appeared and the octahedral size decreased rapidly as the nanorods become longer. The nanorod length reached about $100 \mathrm{~nm}$ with the diameter remaining constant at $10 \mathrm{~nm}$, while the octahedral size decreased to $100 \mathrm{~nm}$. The morphology evolution can also be illustrated by the HR-TEM images (see the Fig. 1(A)-(C)), and the results are consistent with the FE-SEM observation.

The effects of different amounts of doping and different reaction temperatures on morphology were also analyzed by FE-SEM images. Fig. S1 shows that the morphology alters with variation of dopant content. With increase in the amount of $\left.\mathrm{Y}_{(\mathrm{NO}}\right)_{3}$, the shape of as-fabricated samples did not change noticeably. The morphology and size of products maintain the hedgehog-like hierarchical structure. In other words, the amount of dopant has no effect on the morphology and size control. On the other hand, the morphology clearly changed with change in the reaction temperature. Fig. S2 (a) demonstrates that only octahedral particles existed at $150{ }^{\circ} \mathrm{C}$ and these particles aggregated due to no addition of a surfactant. When the reaction temperature was below $150{ }^{\circ} \mathrm{C}$, there was no precipitation from the solution. With increase in the reaction temperature, some short nanoroads appeared on the octahedral surfaces (see Fig. S2 (b)) and the nanorods were not evenly distributed on the surface. There were some rods on one plane, while there was no nanorod on another plane. When the reaction temperature reached $200{ }^{\circ} \mathrm{C}$, many more nanorods emerged, while the octahedral size decreased dramatically and the rods became longer. Fig. S2 (d) indicates that with further increased temperature only the nanorods can be obtained at the cost of consuming $\mathrm{CeO}_{2}$ octahedral completely.

Through systematic experiments, it was shown that the hydrothermal temperature and amount of original sodium phosphate play a vital role in control of $\mathrm{CeO}_{2}$ nanostructures. The as-fabricated samples mainly showed octahedral morphology when $\mathrm{PO}_{4}{ }^{3+}$ concentration was below $0.02 \mathrm{mM}$ and hydrothermal temperature was under $150{ }^{\circ} \mathrm{C}$ due to phosphate group might control the morphology by affect the surface electrostatics potential and energy of octahedral $\mathrm{CeO}_{2}$. A high concentration of $\mathrm{PO}_{4}{ }^{3+}$ will provide more electrostatic potential [23] and a high temperature will supply surface energy on $\mathrm{CeO}_{2}$ surfaces, resulting in the formation of hedgehog-like hierarchical structure $[24,25]$. When the phosphate concentration exceed $0.85 \mathrm{mM}$ and the temperature was higher than $250{ }^{\circ} \mathrm{C}$, we could easily obtain a nanorod $\mathrm{CeO}_{2}$ morphology which 
could be ascribed the following two reasons. First, excessive phosphate groups may restrict the formation of octahedral by steric influence [26]; The second, high temperature will conducive to the formation of nanorods.

\subsection{UV-vis diffuse reflectance spectra and band gap energy}

The UV-vis diffuse reflectance spectra of as-fabricated samples are shown in Fig. 5 (a). The optical band gap energy (Eg) can be calculated from the plot between $E=1240 / \lambda(\mathrm{nm})[27,28]$, where $\lambda$ is the wavelength corresponding to the absorption, and $[\mathrm{F}(\mathrm{R}) \mathrm{h} v]^{1 / 2}[29,30]$ as shown in Fig. 5 (b). $\mathrm{CeO}_{2}$ without doping has absorption at $\sim 380 \mathrm{~nm}$ and band gap energy at $3.20 \mathrm{eV}$, which originate from the charge-transfer between $\mathrm{O}_{2 p}$ and $\mathrm{Ce}_{4 \mathrm{f}}$ states in $\mathrm{O}^{2-}$ and $\mathrm{Ce}^{4+}$. Compared with pure $\mathrm{CeO}_{2}$, the reflectance decreases and absorption red-shifts after the lattice cerium ions have been substituted by yttrium, indicating that the oxygen vacancies increased due to a charge compensation mechanism [31]. Fig. 5 (b) clearly reveals that pure $\mathrm{CeO}_{2}$ exhibits low band gap energy compared to that of yttrium-doped ceria, and the Eg values are $3.20 \mathrm{eV}$ and $\sim 3.35 \mathrm{eV}$, respectively. Subtle differences of band gap between various morphologies of yttrium-doped ceria were detected from the plot of K-M curves. Fig. 5(b) indicates that the octahedral morphology has a higher Eg value than the values of other shapes of ceria doped by yttrium. This result is consisted with the results of Raman analysis, and this optical property is very important for good photocatalytic performance.

\subsection{Photocatalytic activity for acetaldehyde decomposition}

The photocatalytic activities of as-fabricated products were evaluated by measuring $\mathrm{CO}_{2}$ evolution form the photocatalytic decomposition of acetaldehyde.

Fig. 6 (a) shows the time course of $\mathrm{CO}_{2}$ liberation of samples with different amounts of original materials $\mathrm{Na}_{3} \mathrm{PO}_{4}$. Photocatalytic activities were enhanced by increasing the amount of $\mathrm{Na}_{3} \mathrm{PO}_{4}$ at a low concentration, and photocatalytic activity at $0.47 \mathrm{mM} \mathrm{Na}_{3} \mathrm{PO}_{4}$ was higher than that of other concentrations whit further increase in the amount of $\mathrm{Na}_{3} \mathrm{PO}_{4}$, the photocatalytic activities decreased. Change in photocatalytic activities with change in the amount of $\mathrm{Na}_{3} \mathrm{PO}_{4}$ can be attributed to the change in morphology. Only the sample with a hedgehog-like morphology had a higher concentration of oxygen vacancies than those of other morphology products owing to the hedgehog-like three dimensional hierarchical structure has a larger specific surface area (see the Table 1), and more activity oxygen exists on higher specific surface area.

Fig. 6 (b) shows the time course of $\mathrm{CO}_{2}$ liberation of samples with different reaction temperatures. The sample as-fabricated at $200{ }^{\circ} \mathrm{C}$ exhibited the highest photocatalytic activities. Photocatalytic activities change trend with reaction temperature is similar to the influence of content $\mathrm{Na}_{3} \mathrm{PO}_{4}$ which is attributed to morphology evolution. That means at higher $\left(>250{ }^{\circ} \mathrm{C}\right)$ and lower $\left(<170{ }^{\circ} \mathrm{C}\right)$ reaction temperatures purely $1 \mathrm{D}$ structure, such as octahedral and nanorod, can be obtained. Compared with the photocatalytic activities of samples with a hedgehog-like 3D hierarchical structure, these samples with 1D morphology shown low photocatalytic activities owing to the lower concentration of oxygen vacancies and smaller $\mathrm{S}_{\mathrm{BET}}$.

Fig. 6 (c) shows the time course of $\mathrm{CO}_{2}$ liberation of samples with different of dopant concentrations. When dopant concentration is low $(<0.375 \mathrm{mmol})$, the as-fabiracted samples show a low $\mathrm{CO}_{2}$ liberation rate as in the case of pure $\mathrm{CeO}_{2}$. However, with increase in the concentration

of the dopant, the $\mathrm{CO}_{2}$ liberation rate rapidly improved, which demonstrated that a high content 
dopant is beneficial for enhancement of photocatalytic activities. When the content of dopant reached $0.747 \mathrm{mmol}$, the fabricated sample showed the highest $\mathrm{CO}_{2}$ liberation rate, and with further increase in concentration of the dopant, the $\mathrm{CO}_{2}$ liberation rate decreased.

In order to investigate the photocatalytic reusability and stability of as-fabricated yttrium-doped hedgehog-like $\mathrm{CeO}_{2}$, a periodic photocatalytic decomposition experiments were performed. Fig S3 illustrates cycling runs of photocatalytic activity evaluation. The photocatalytic performance of sample has no detectable loss after four recycles and the $\mathrm{CO}_{2}$ generation rate does not change obviously. Meanwhile, ICP-AES measurement indicated that yttrium doped on $\mathrm{CeO}_{2}$ can hardly be leached out in suspensions even by 48 hours vigorous stirred (see the Fig S4). Therefore, we can draw a conclusion that yttrium doped $\mathrm{CeO}_{2}$ with 3D hedgehog-like hierarchical structure possess good photocatalytic reusability as well as excellent stability.

Oxygen vacancy concentrations of the products play the predominant role in governing the trend of photocatalytic activities. Regardless of the 1D octahedral, nanorod or 3D hedgehog-like hierarchical structure and regardless of the amount of the dopant, the ultimate goal is to control the oxygen vacancy concentrations in the samples. When fabricated samples are irradiated under UV light, electrons are excited from the $\mathrm{O}_{2 p}$ valence band to the conduction band $\left(\mathrm{Ce}_{4 \mathrm{f}}\right)$ resulting in the formation of hole and electron pairs [32], and the holes easily get trapped on the oxygen ions $[33,34]$. When the number of oxygen vacancies is higher, the oxygen ion mobility becomes higher [35]. Transportation mobility of lattice oxygen ions is beneficial for the separation of photogenerated electrons and holes. Overall, the photogenerated electron and holes recombination will be restricted in higher oxygen vacancies concentration. At the same time, different exposed crystal plane also influence the photocatalytic activity. Traditional $\mathrm{CeO}_{2}$ with octahedral morphology are enclosed by eight (111) planes as well as the nanorod shape are enclosed by the (200) and (110) planes. Combined with previous studies [36, 37], $\mathrm{CeO}_{2}$ with nanorod morphology predominantly exposed (200) plane shows higher photocatalytic activity than $\mathrm{CeO}_{2}$ with octahedral exposed stable (111) plane attributed to the (200) plane are unstable and showing higher surface energy. Hence, 3D hedgehog-like hierarchical structure is beneficial for the formation of a high concentration of oxygen vacancies and has been shown to possess the best photocatalytic activity.

\section{Conclusion}

In summary, $\mathrm{CeO}_{2}$ with yttrium-doped hedgehog-like hierarchical architectures was prepared successfully by a simple hydrothermal method. The optimum experimental conditions for synthesis of octahedral plus nanorod $\mathrm{CeO}_{2}$ were found to be $0.47 \mathrm{mM} \mathrm{Na}_{3} \mathrm{PO}_{4}$ and $0.747 \mathrm{mmol}$ $\mathrm{Y}\left(\mathrm{NO}_{3}\right)_{3}$ as original materials and reaction at $200{ }^{\circ} \mathrm{C}$ for 24 hours. The method of synthetic is effective and reproducible and can be further expanded to fabricate other rare-earth-doped morphology-controlled inorganic nanoparticle materials. Hedgehog-like yttrium-doped $\mathrm{CeO}_{2}$ exhibits an advantage of photocatalytic activity. The results of activity evaluation are consistent with the results of Raman, UV-vis DRS, XRD and SEM analyses. The trend for changes in photocatalytic activities was explained by the oxygen vacancies derived from changes in morphology and yttrium doping. Consequently, yttrium-doped hedgehog-like hierarchical $\mathrm{CeO}_{2}$ is a promising material for practical application in photocatalytic materials and SOFCs as well as other novel environmental friendly materials. 


\section{Acknowledgement}

This work was supported by the JST PRESTO program and JST ACT-C program. The authors are grateful for the financial support to the National Natural Science Foundation of China (No. 50873085) 


\section{Figure captions}

Fig. 1. XRD patterns of as-fabricated samples.

Fig. 2. Raman spectra of as-fabricated samples.

Fig. 3. FE-SEM and HR-TEM images of as-fabricated samples at different concentrations of $\mathrm{Na}_{3} \mathrm{PO}_{4}$ : (a)-(A) $0.20 \mathrm{mM}$, (b)-(B) $0.47 \mathrm{mM}$ and (c)-(C) $0.85 \mathrm{mM}$.

Fig. 4. HAADF-STEM and EDS mapping images of as-fabricated samples.

Fig.5. Uv-vis diffuse reflectance spectra of samples with different morphology.

Fig.6. Time course of $\mathrm{CO}_{2}$ liberation from acetaldehyde decomposition of as-fabricated samples.

Table 1. Relevant data of as-fabircated samples used in the work.

Fig. S1. FE-SEM images of as-fabricated samples at different dopant contents: (a)0.05 mmol, (b) $0.102 \mathrm{mmol}$, (c) $0.375 \mathrm{mmol}$, (d) $0.747 \mathrm{mmol}$, (e) $0.91 \mathrm{mmol}$ and (f) $1.2 \mathrm{mmol}$.

Fig. S2. FE-SEM images of as-fabricated samples at different reaction temperature: (a) $150{ }^{0} \mathrm{C}$, (b) $170{ }^{\circ} \mathrm{C}$, (c) $200{ }^{\circ} \mathrm{C}$ and (d) $250{ }^{\circ} \mathrm{C}$.

Fig. S3. cycling runs of photocatalytic activity evaluation of yttrium doped $\mathrm{CeO}_{2}$ with $3 \mathrm{D}$ hedgehog-like hierarchical structure.

Fig.S4. the yttrium elution variation curve of yttrium doped $\mathrm{CeO}_{2}$ with $3 \mathrm{D}$ hedgehog-like hierarchical structure. 


\section{References:}

[1] K. Zhou, Z. Yang, S. Yang, Chemistry of Materials 19 (2007) 1215-1217.

[2] H. Imagawa, A. Suda, K. Yamamura, S. Sun, The Journal of Physical Chemistry C 115 (2011) 1740-1745.

[3] L. Yan, R. Yu, J. Chen, X. Xing, Crystal Growth \& Design 8 (2008) 1474-1477.

[4] R.K. Pati, I.C. Lee, K.J. Gaskell, S.H. Ehrman, Langmuir 25 (2008) 67-70.

[5] Z.-L. Wang, G.-R. Li, Y.-N. Ou, Z.-P. Feng, D.-L. Qu, Y.-X. Tong, The Journal of Physical Chemistry C 115 (2010) 351-356.

[6] Z. Wang, Z. Quan, J. Lin, Inorganic Chemistry 46 (2007) 5237-5242.

[7] S. Kitano, N. Murakami, T. Ohno, Y. Mitani, Y. Nosaka, H. Asakura, K. Teramura, T. Tanaka,

H. Tada, K. Hashimoto, H. Kominami, The Journal of Physical Chemistry C 117 (2013) 11008-11016.

[8] C. Paun, O.V. Safonova, J. Szlachetko, P.M. Abdala, M. Nachtegaal, J. Sa, E. Kleymenov, A. Cervellino, F. Krumeich, J.A. van Bokhoven, The Journal of Physical Chemistry C 116 (2012) $7312-7317$.

[9] M. Jobbágy, F. Mariño, B. Schönbrod, G. Baronetti, M. Laborde, Chemistry of Materials 18 (2006) 1945-1950.

[10] H. Xiao, Z. Ai, L. Zhang, The Journal of Physical Chemistry C 113 (2009) 16625-16630.

[11] F. Dvořák, O. Stetsovych, M. Steger, E. Cherradi, I. Matolínová, N. Tsud, M. Škoda, T. Skála, J. Mysliveček, V. Matolín, The Journal of Physical Chemistry C 115 (2011) 7496-7503.

[12] Q. Zhang, B. Xu, S. Yuan, M. Zhang, T. Ohno, Materials Letters 121 (2014) 109-112.

[13] Z. Yang, D. Han, D. Ma, H. Liang, L. Liu, Y. Yang, Crystal Growth \& Design 10 (2009) 291-295.

[14] L.-W. Qian, X. Wang, H.-G. Zheng, Crystal Growth \& Design 12 (2011) 271-280.

[15] S.L. Mitchell, J. Guzman, Materials Chemistry and Physics 114 (2009) 462-466.

[16] L.-S. Zhong, J.-S. Hu, A.-M. Cao, Q. Liu, W.-G. Song, L.-J. Wan, Chemistry of Materials 19 (2007) $1648-1655$.

[17] Z.-Y. Pu, X.-S. Liu, A.-P. Jia, Y.-L. Xie, J.-Q. Lu, M.-F. Luo, The Journal of Physical Chemistry C 112 (2008) 15045-15051.

[18] A.E. Baranchikov, O.S. Polezhaeva, V.K. Ivanov, Y.D. Tretyakov, CrystEngComm 12 (2010) 3531.

[19] C.-Y. Cao, Z.-M. Cui, C.-Q. Chen, W.-G. Song, W. Cai, The Journal of Physical Chemistry C 114 (2010) 9865-9870.

[20] Y. Lee, G. He, A.J. Akey, R. Si, M. Flytzani-Stephanopoulos, I.P. Herman, Journal of the American Chemical Society 133 (2011) 12952-12955.

[21] S. Chang, M. Li, Q. Hua, L. Zhang, Y. Ma, B. Ye, W. Huang, Journal of Catalysis 293 (2012) $195-204$

[22]B.M. Reddy, A. Khan, Y. Yamada, T. Kobayashi, S. Loridant, J.-C. Volta, Langmuir 19 (2003) 3025-3030

[23] G. Cornelis, B. Ryan, M.J. McLaughlin, J.K. Kirby, D. Beak, D. Chittleborough, Environmental Science \& Technology 45 (2011) 2777-2782.

[24] D.R. Mullins, P.M. Albrecht, T.-L. Chen, F.C. Calaza, M.D. Biegalski, H.M. Christen, S.H. Overbury, The Journal of Physical Chemistry C 116 (2012) 19419-19428.

[25] Z.L. Wang, X. Feng, The Journal of Physical Chemistry B 107 (2003) 13563-13566. 
[26] M. Lin, Z.Y. Fu, H.R. Tan, J.P.Y. Tan, S.C. Ng, E. Teo, Crystal Growth \& Design 12 (2012) 3296-3303.

[27] P. Ji, J. Zhang, F. Chen, M. Anpo, The Journal of Physical Chemistry C 112 (2008) 17809-17813.

[28] J. Li, G. Lu, H. Li, Y. Wang, Y. Guo, Y. Guo, Journal of Colloid and Interface Science 360 (2011) 93-99.

[29] Y. Park, S.K. Kim, D. Pradhan, Y. Sohn, Chemical Engineering Journal 250 (2014) 25-34.

[30] C. Karunakaran, P. Gomathisankar, ACS Sustainable Chemistry \& Engineering 1 (2013) $1555-1563$.

[31] J. Wang, D.N. Tafen, J.P. Lewis, Z. Hong, A. Manivannan, M. Zhi, M. Li, N. Wu, Journal of the American Chemical Society 131 (2009) 12290-12297.

[32] M.a.D. Hernández-Alonso, A.B. Hungría, A. Martínez-Arias, M. Fernández-García, J.M. Coronado, J.C. Conesa, J. Soria, Applied Catalysis B: Environmental 50 (2004) 167-175.

[33] P. Bera, A. Gayen, M.S. Hegde, N.P. Lalla, L. Spadaro, F. Frusteri, F. Arena, The Journal of Physical Chemistry B 107 (2003) 6122-6130.

[34] M.B. Watkins, A.S. Foster, A.L. Shluger, The Journal of Physical Chemistry C 111 (2007) 15337-15341.

[35] B. Murugan, A.V. Ramaswamy, The Journal of Physical Chemistry C 112 (2008) 20429-20442.

[36] K. Zhou, X. Wang, X. Sun, Q. Peng, Y. Li, Journal of Catalysis 229 (2005) 206-212.

[37] Tana, M. Zhang, J. Li, H. Li, Y. Li, W. Shen, Catalysis Today 148 (2009) 179-183. 


\section{Table 1}

\begin{tabular}{|c|c|c|c|c|}
\hline sample & $\begin{array}{c}\text { Lattice a } \\
\text { Value }(\AA) \\
\end{array}$ & $\begin{array}{c}\text { Particles size } \\
(\mathbf{n m})\end{array}$ & $\begin{array}{c}\mathbf{R}_{\text {dopant }} / \mathbf{F 2 g} \\
(\%) \\
\end{array}$ & $\mathrm{S}_{\mathrm{BET}}\left(\mathrm{m}^{2} / \mathrm{g}\right)$ \\
\hline Pure $\mathrm{CeO}_{2}$ & 5.41001 & 55.987 & 0 & 8.1 \\
\hline $\begin{array}{l}\mathrm{CeO}_{2}+\mathrm{Y} / \\
\text { octahedral }\end{array}$ & 5.33052 & 48.162 & 4.16 & 23.6 \\
\hline $\begin{array}{c}\mathrm{CeO}_{2}+\mathrm{Y} / \\
\text { octahedral+nanrod }\end{array}$ & 5.31606 & 28.831 & 4.93 & 70.4 \\
\hline $\begin{array}{c}\mathrm{CeO}_{2}+\mathrm{Y} / \\
\text { nanorod }\end{array}$ & 5.27510 & 32.833 & 3.62 & 49.1 \\
\hline
\end{tabular}


Fig. 1
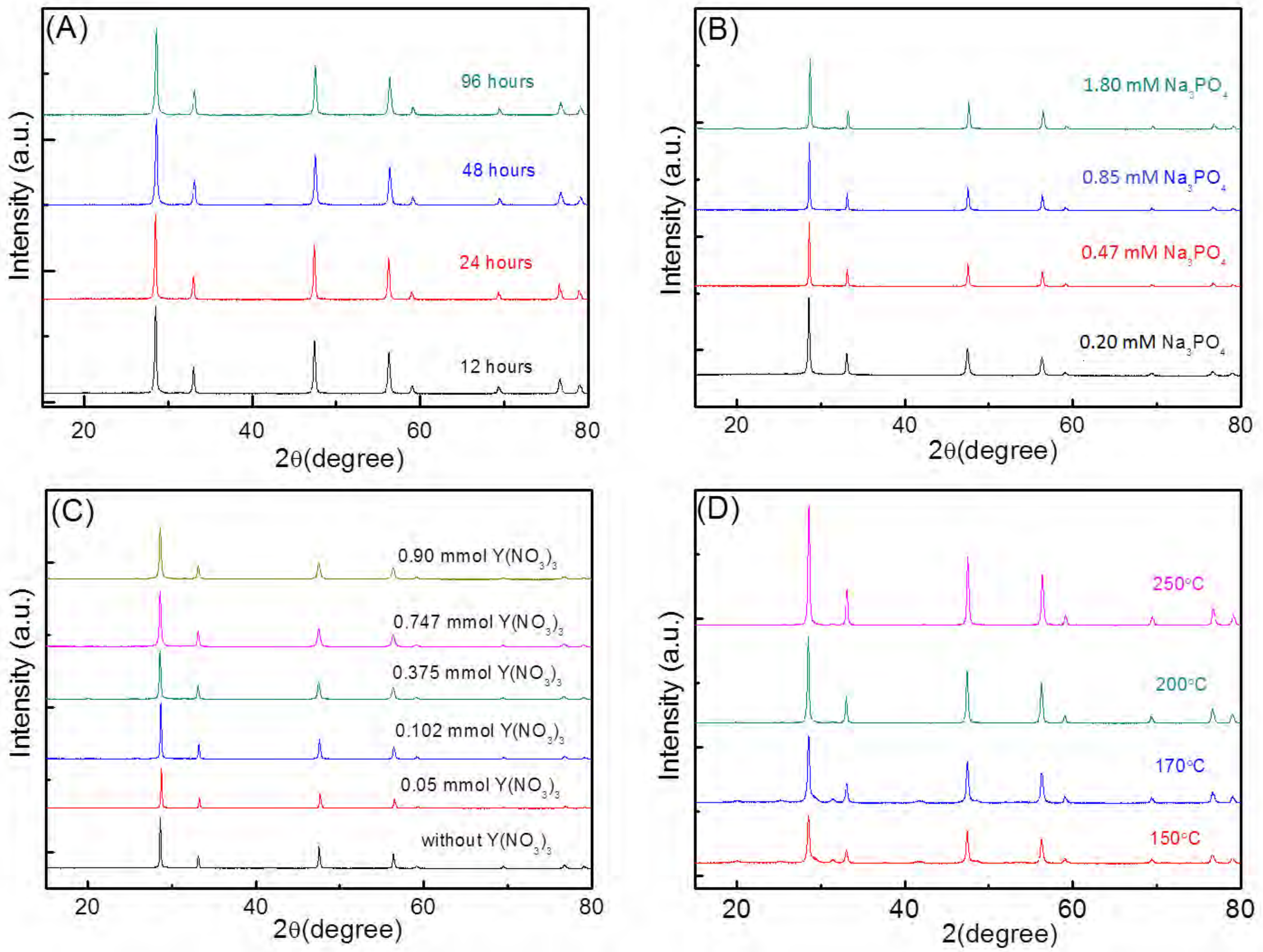
Fig. 2
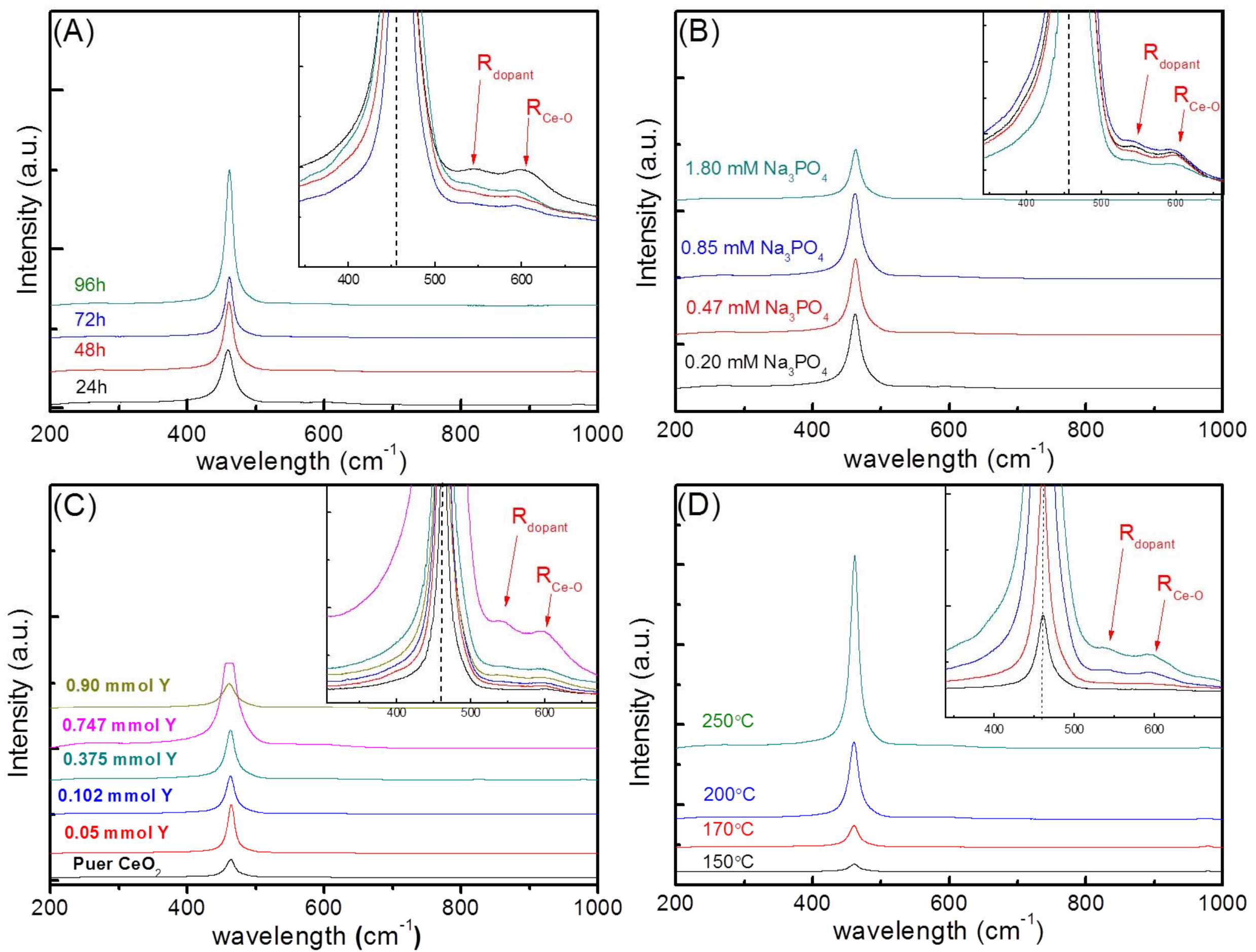
Fig. 3
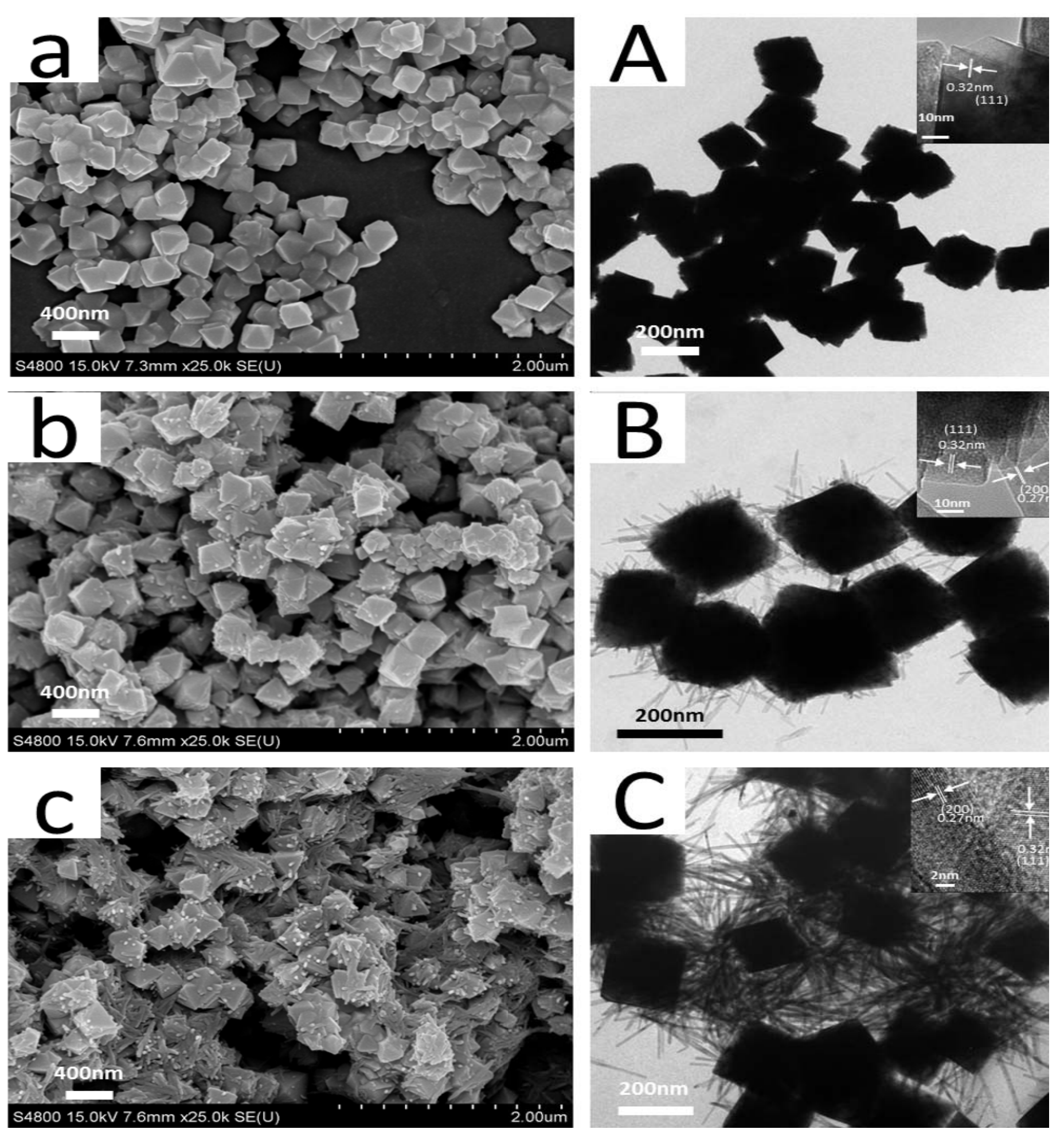
Fig. 4

AADF

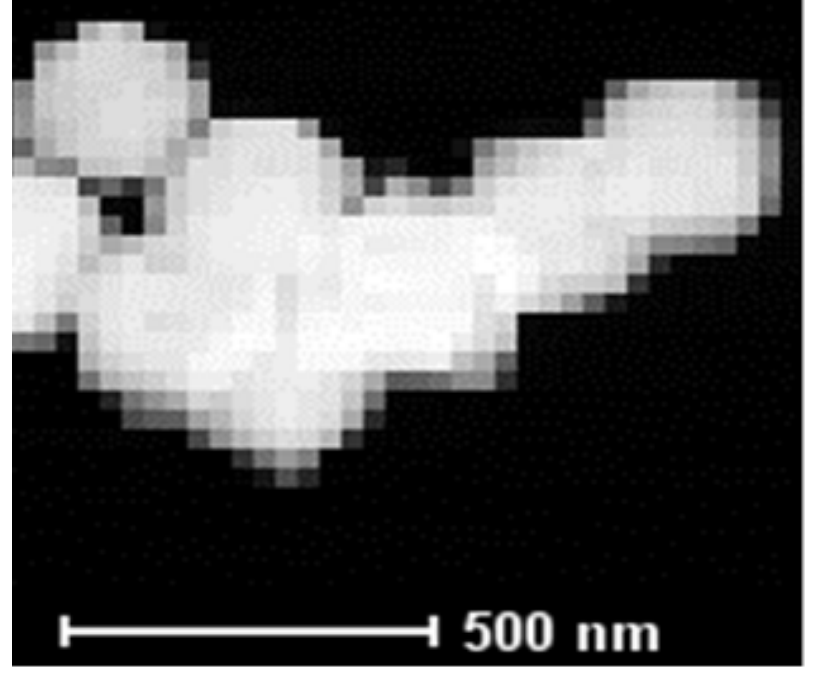

\section{Ce-K}

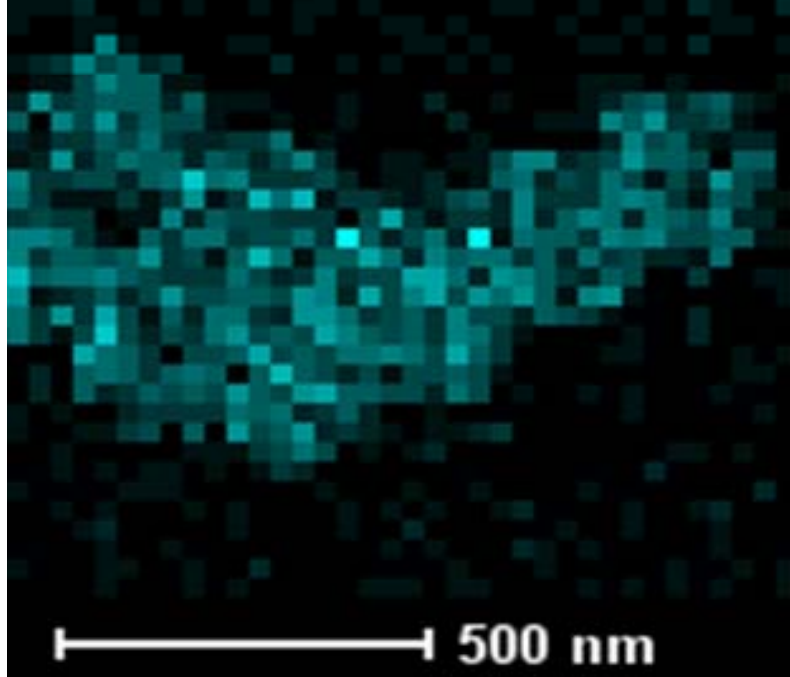

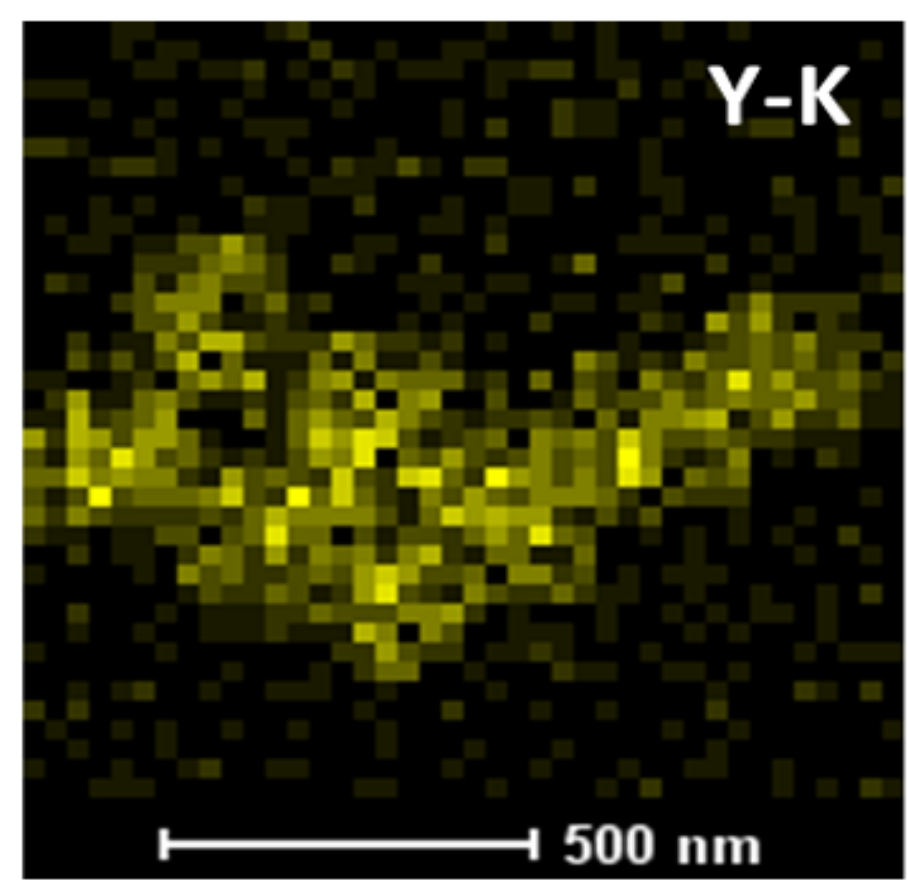

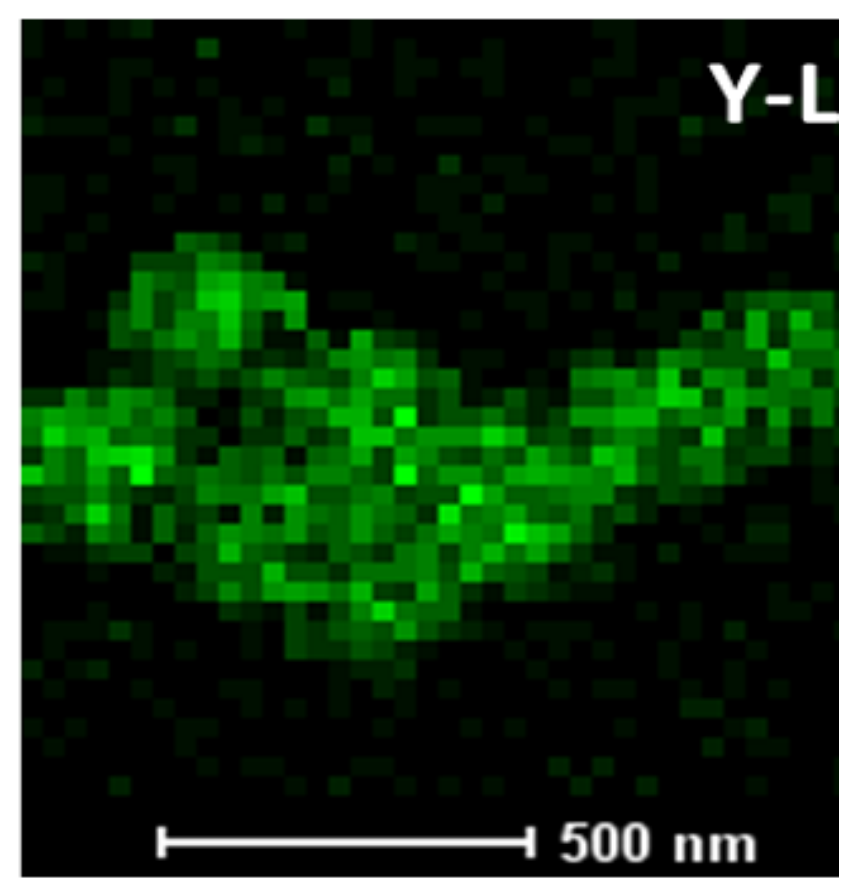

Ce-L

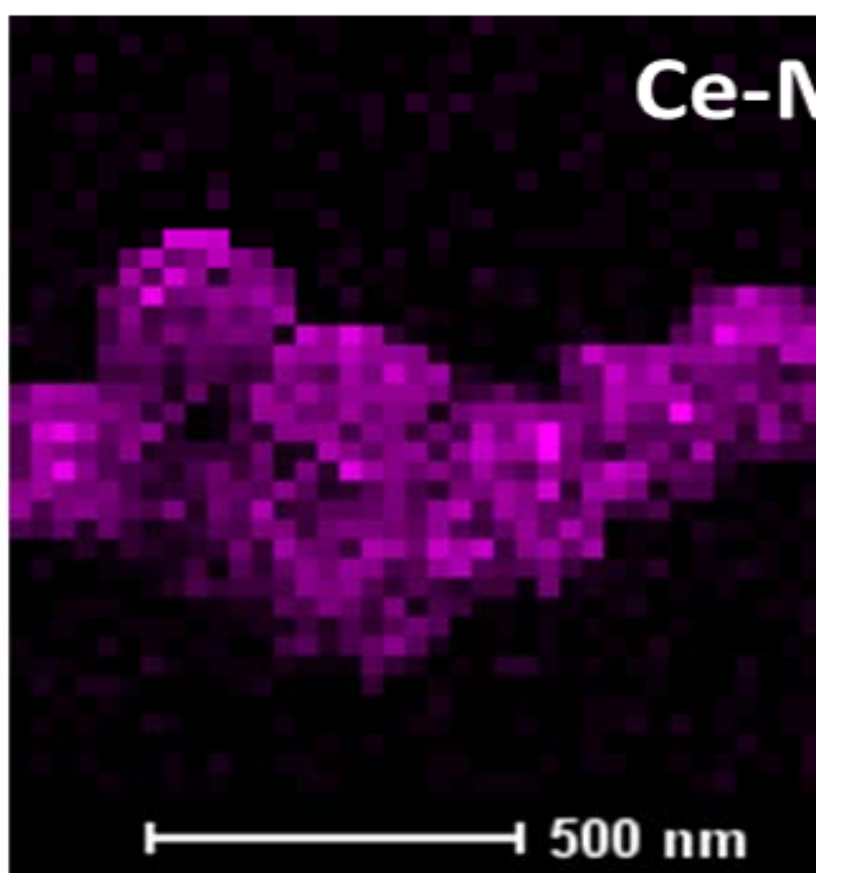


Fig. 5
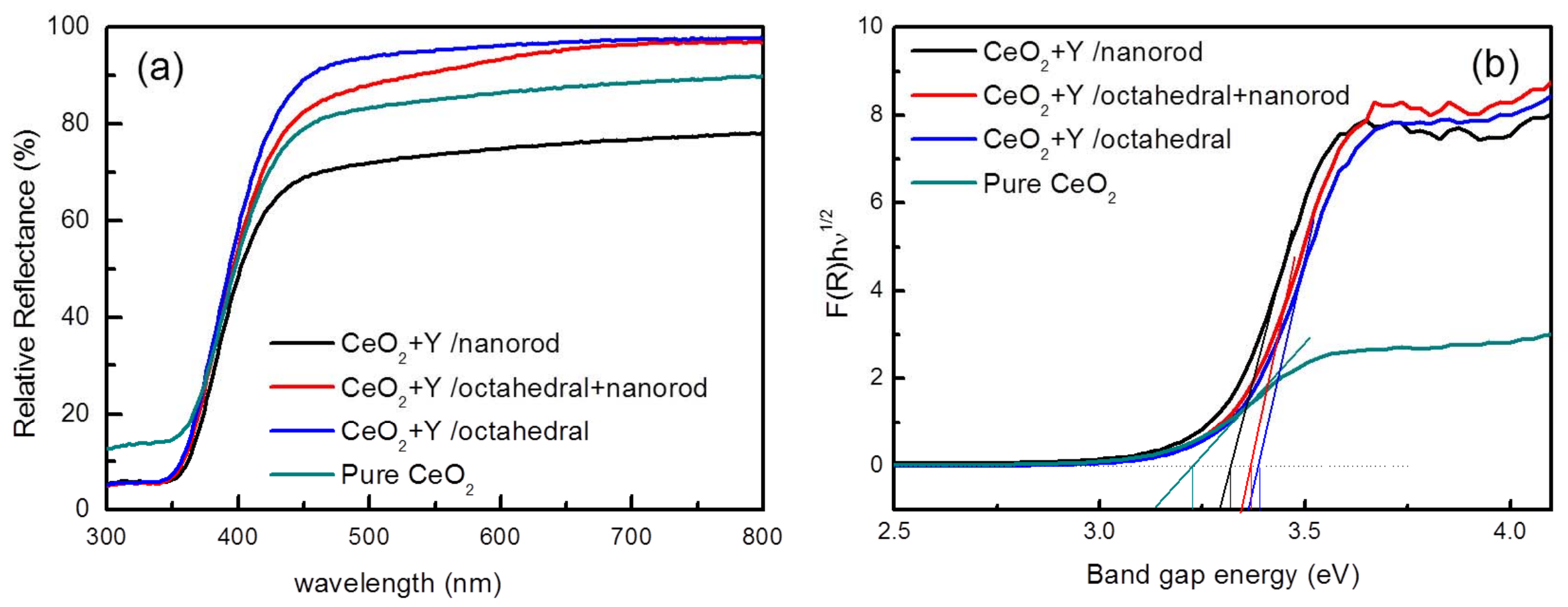
Fig. 6
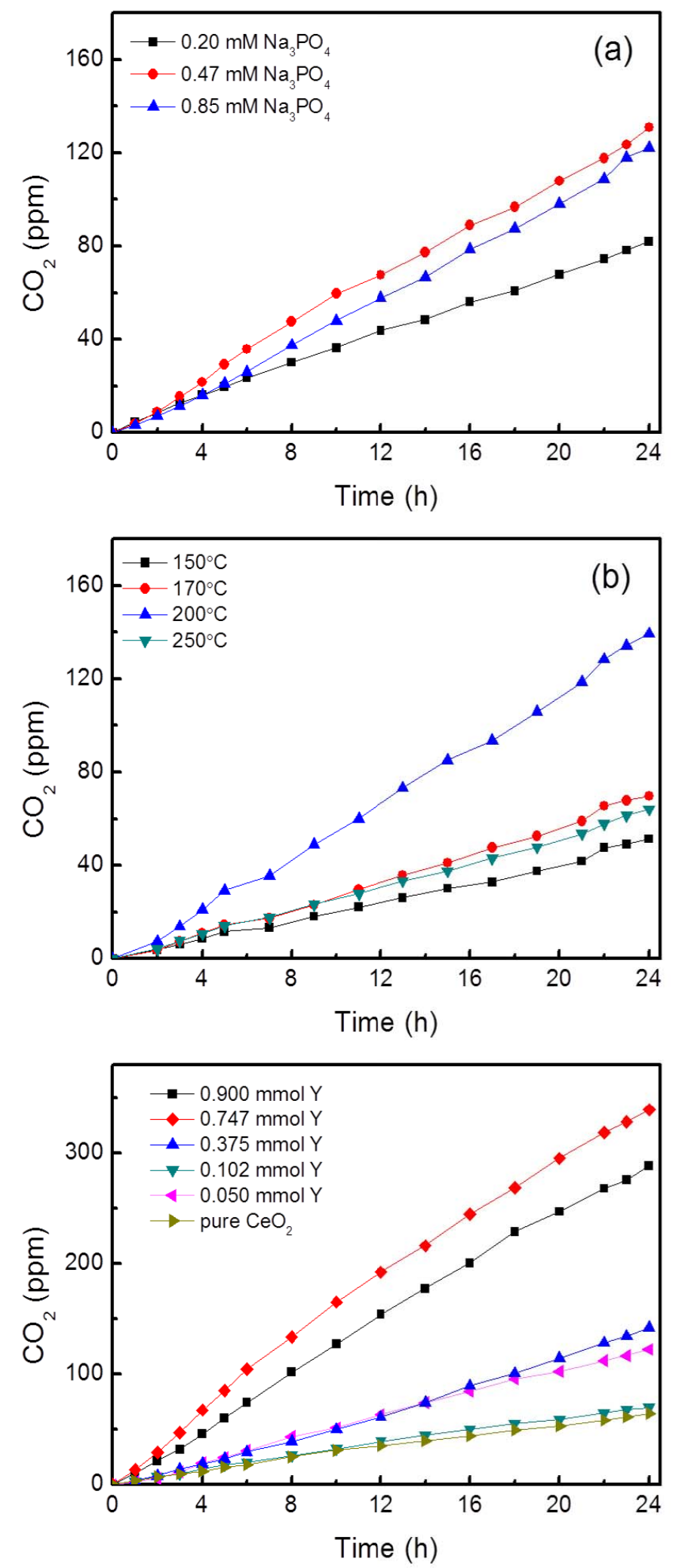
Graphical abstract
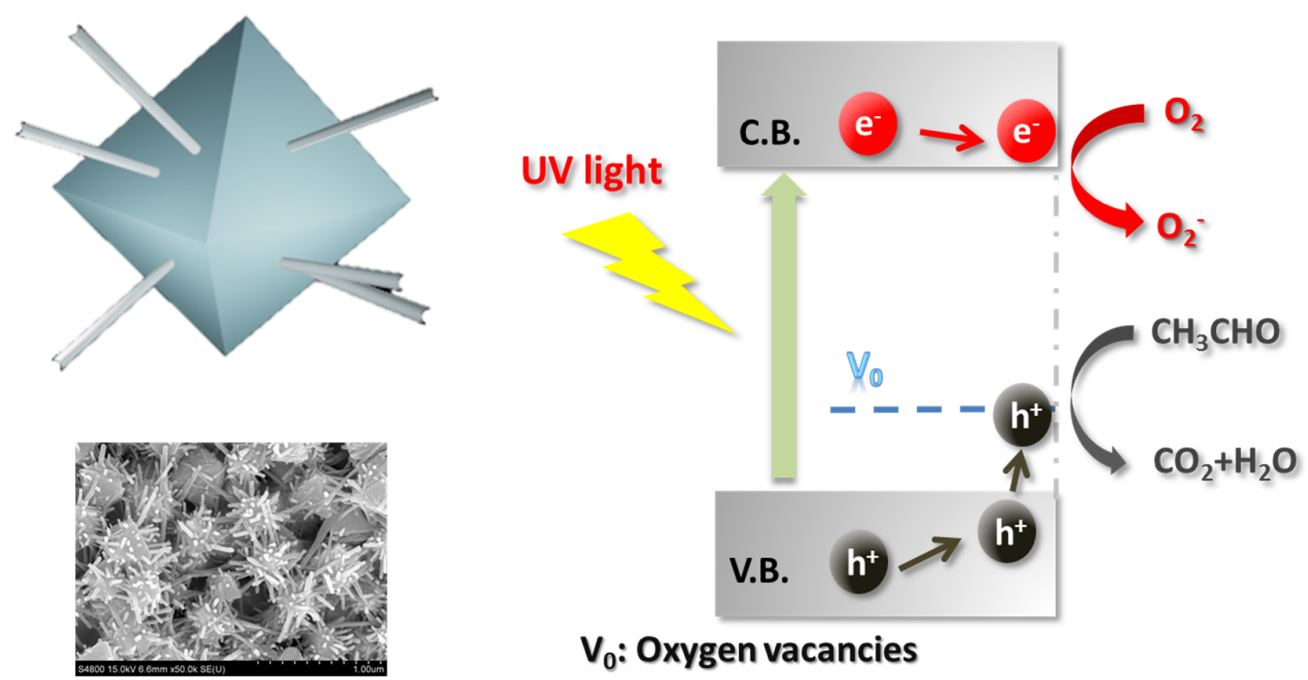